IGNACIO MONTALDO, SILVIA COLOMBO, 2013

\section{Ignacio Montaldo}

Profesor, Universidad de Buenos Aires, Buenos Aires, Argentina

Arquitecto, Universidad de Buenos Aires, 2003. Desde 2004 dirige Ignacio Montaldo Arquitectos y combina su actividad profesional con la académica. En el año 2007 obtuvo el primer premio en la categoría Intervenciones Urbanas del premio Bienal para obras construidas del Colegio de Arquitectos de la Provincia de Buenos Aires y en 2008 obtuvo la primera mención de honor en la Bienal Iberoamericana de Quito en la categoría Arquitectura Paisajista. Ejerció la docencia en la Universidades Torcuato di Tella y Palermo en Buenos Aires: actualmente es profesor en las universidades de Buenos Aires y de General de San Martín.

\section{Silvia Colombo}

Arquitecta independiente, Buenos Aires, Argentina.

Arquitecta, Universidad de Buenos Aires, 2000. Se ha desempeñado como docente en la Universidad de Buenos Aires y en la Universidad de Palermo. En 2002 fue finalista en el concurso internacional de intercambio docente en The University of Illinois at Chicago. Desde 2005 desarrolla actividad profesional independiente dentro de los ámbitos público y privado, en proyectos de variadas escalas. Su trabajo ha obtenido diversos premios en concursos de arquitectura.

Esta es una operación arquitectónica realizada entre los volúmenes construidos: tiende un pavimento, dispone luminarias y ajusta perfiles de la calle para definir una nueva realidad y cualificar el espacio público.

Palabras clave: Arquitectura - Argentina, urbanismo Argentina, renovación urbana, mobiliario urbano, plaza, paseo.

This is an intervention on the space in-between buildings: it spreads a pavement, lays out lighting and adjusts the street profiles to define a new reality that qualify public space.

Keywords: Architecture - Argentina, urbanism - Argentina, urban renewal, street furniture, square, walkway.
Este proyecto es parte de una estrategia de intervención y recuperación de los espacios públicos del casco histórico de la ciudad de San Fernando, en la zona norte del Gran Buenos Aires. Los espacios públicos comprendidos en este proyecto son el centro cívico, la plaza Mitre y su centro comercial a cielo abierto, ubicado a lo largo de la calle Constitución. La propuesta se construye desde la importancia del rol social en el espacio público dentro de la trama urbana.

ETAPA 1: PLAZA MITRE

El objetivo central de la intervención es recuperar la calidad ambiental de la plaza Mitre. La estrategia principal consiste en entender la plaza como un recinto espacial único definido por las edificaciones circundantes. La intervención arquitectónica intenta reforzar este concepto a través de la continuidad de nivel de piso entre los sectores de circulación vehicular y peatonal, además de la unificación de materiales y color de solado. Se reordena el estacionamiento en todo el recinto, se disminuye la velocidad de circulación vehicular y se prioriza el uso peatonal.

ETAPA 2：CALLE CONSTITUCIÓN

Desde los años noventa -entre otros motivos por la aparición de los grandes centros comerciales- aquellos emplazados a cielo abierto han experimentado su decadencia. La revitalización del centro comercial de la calle Constitución se proyecta como una intervención urbana destinada a garantizar la vitalidad de la ciudad. El proyecto cuenta con un sistema capaz de operar con pocas variables, pero que obtiene resultados completos.

El nivel de pavimentos vehiculares y peatonales unificados logran una integración del área incorporando ambos usos. Para la calzada vehicular se utilizó pavimento intertrabado de hormigón, mientras que para la vía peatonal se usó mosaico granítico. Si bien se contemplan diferentes materialidades, el punto de unión es el color rojo empleado en ambas estructuras.

Eliminando el área de estacionamiento se redujo la calzada vehicular a un carril y se incrementó la dimensión de las zonas peatonales. El entorno se forestó con dos especies de acacia: fricia y casque rouge. Estos árboles de flor y hoja caduca, brindan sombra en verano y permiten el paso del sol en invierno. Así, el tiempo no solo se considera como una variable, sino que se transforma en el elemento esencial en la recuperación de un espacio público de principios del siglo x IX.

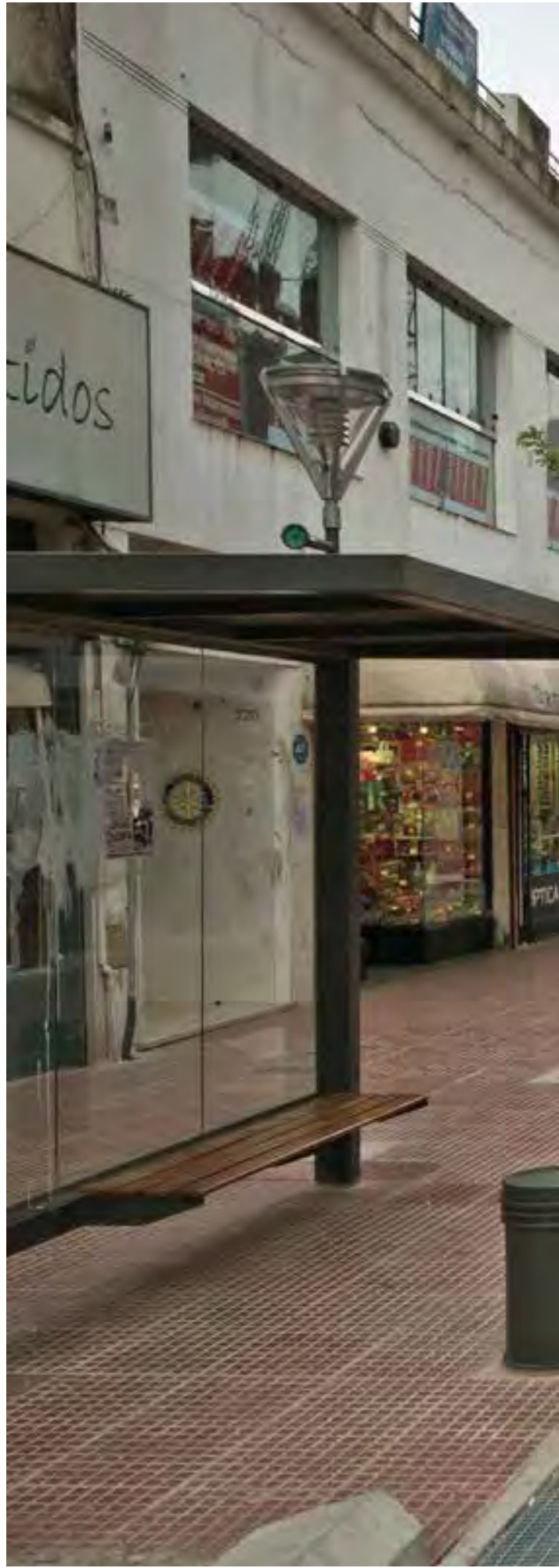


REMODELACIÓN DEL ESPACIO PÚBLICO, SAN FERNANDO, ARGENTINA
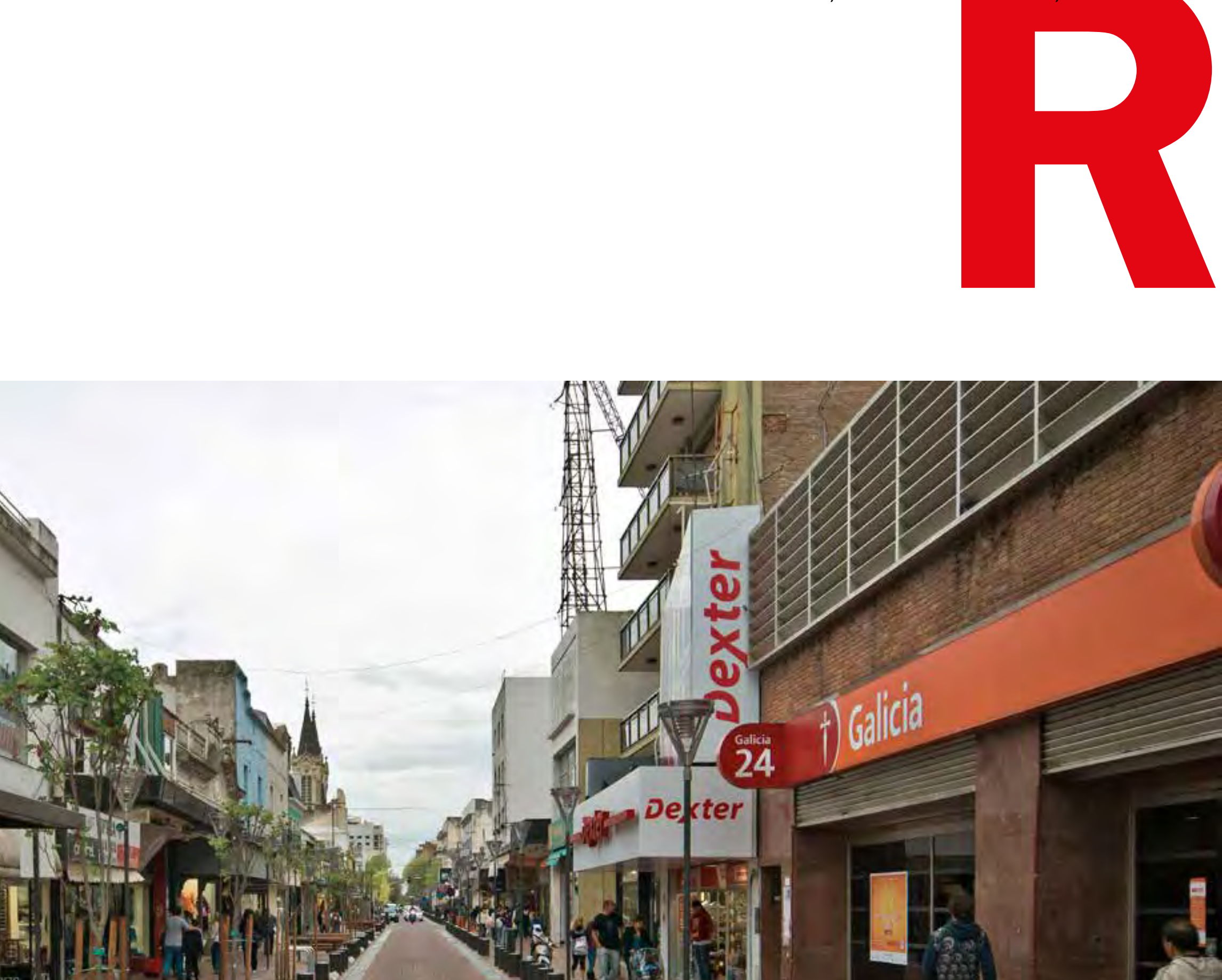

ser:

ax

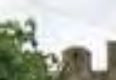

A)

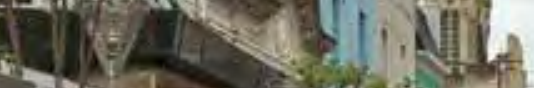

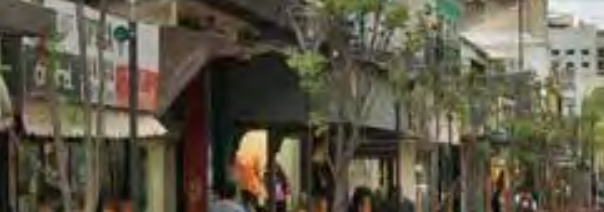
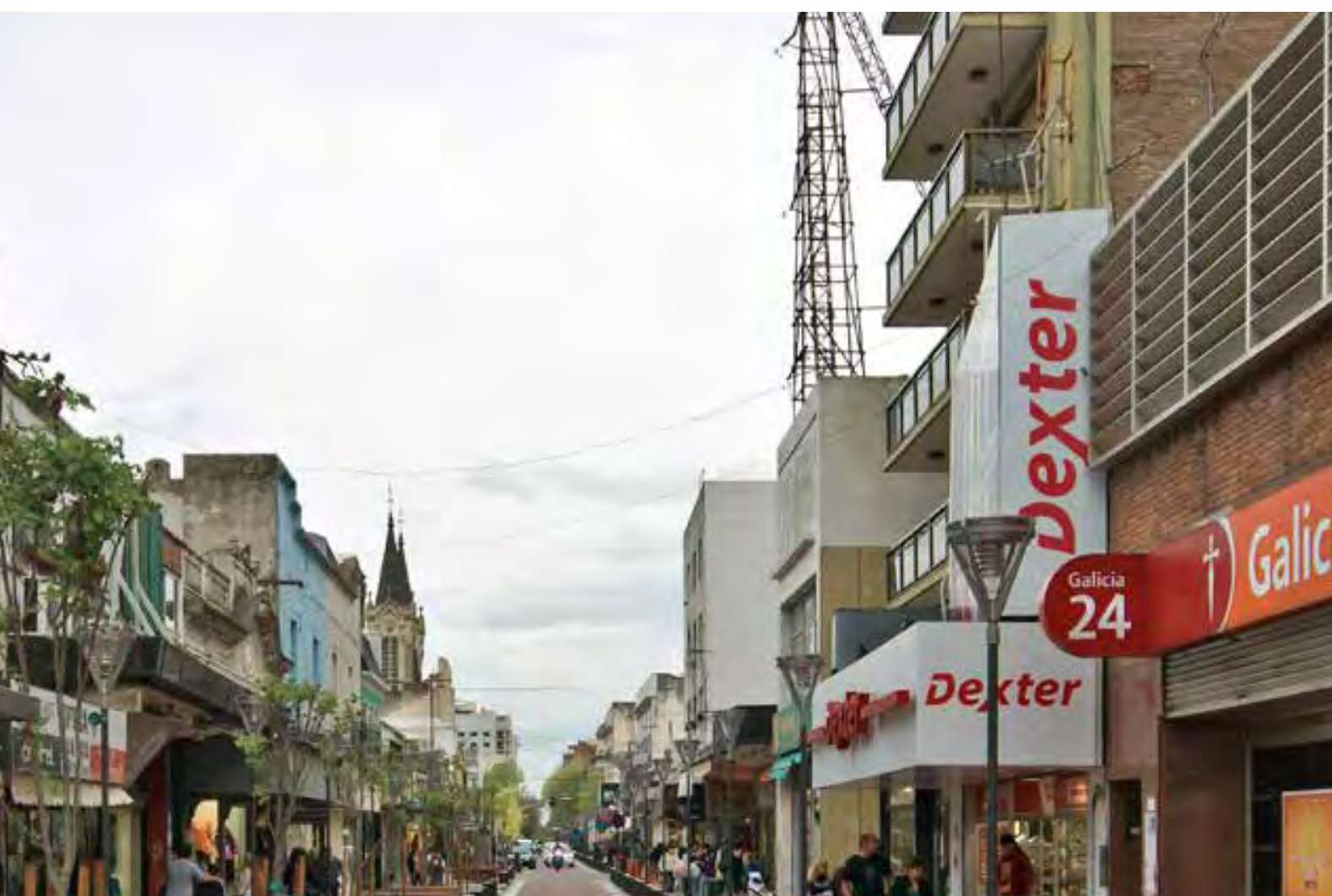

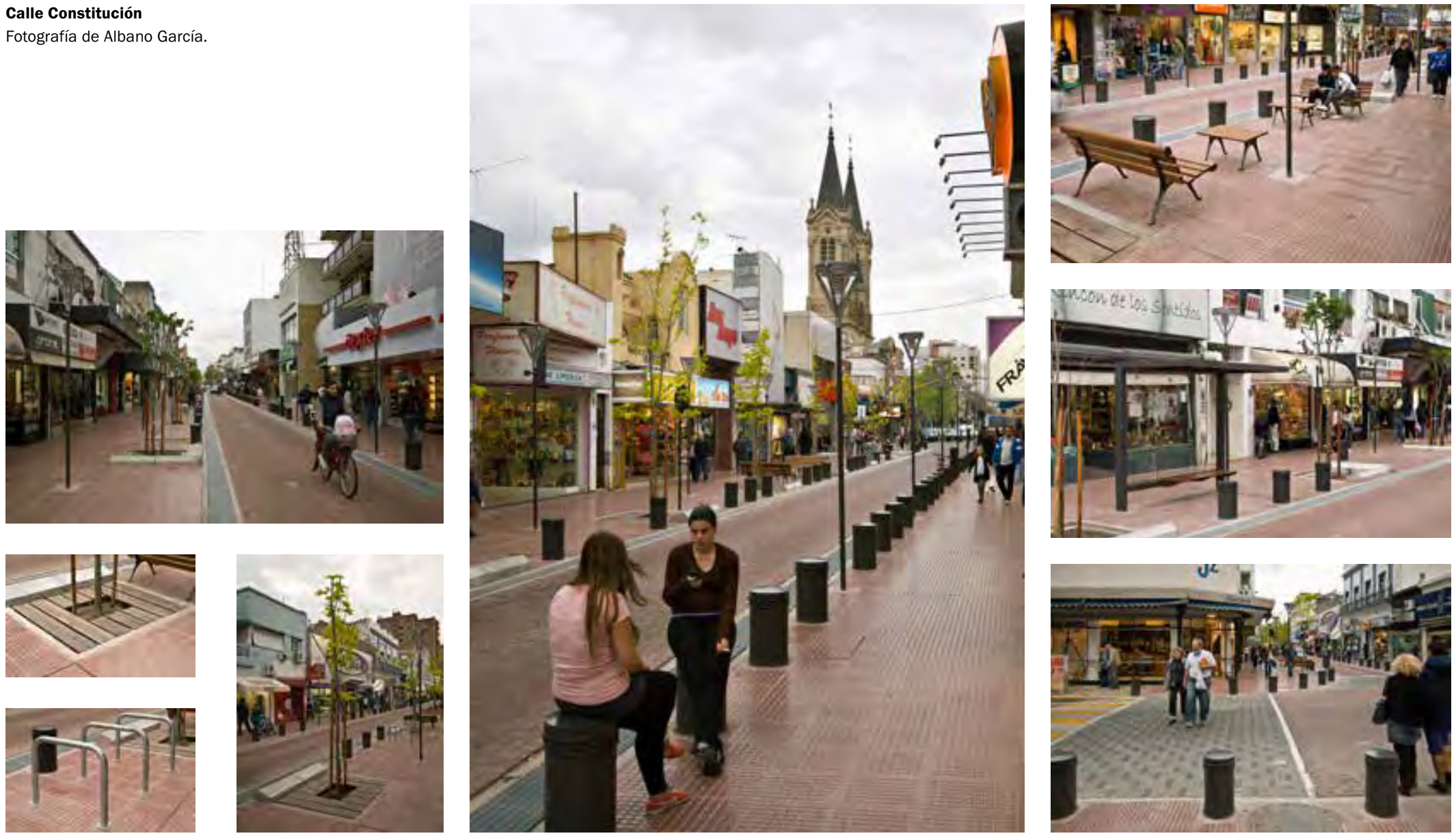

Planta de la intervención
E. 1: 7.500

$\therefore$ \%

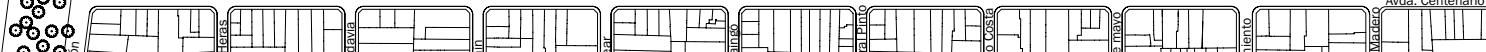
$\therefore \circ: 0$ :

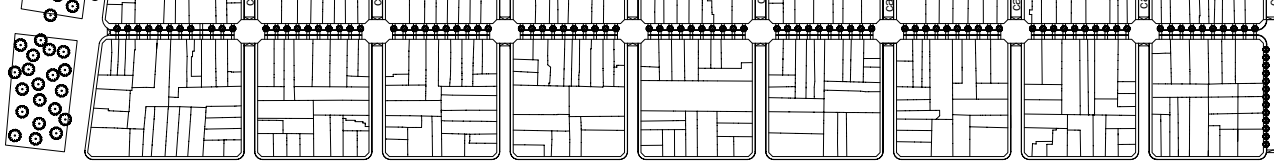

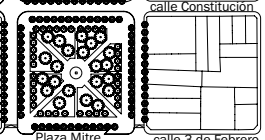

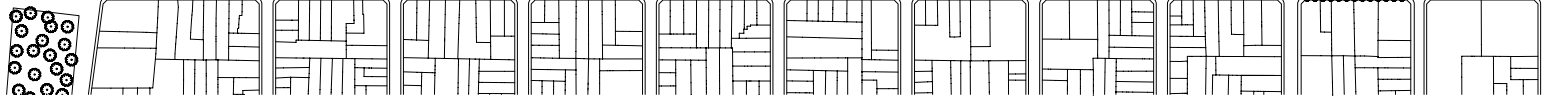

Planta calle Constitución, cuadra tipo

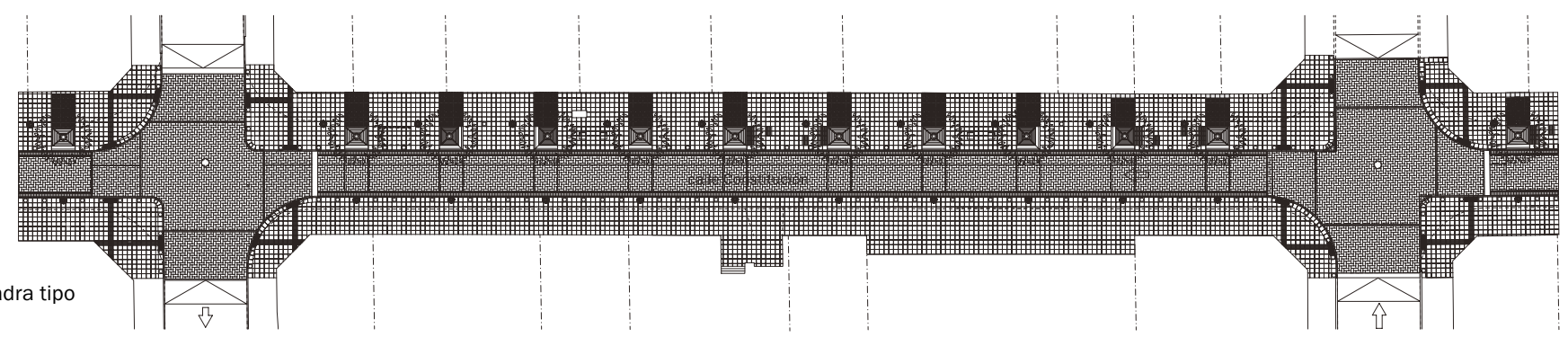

E. 1: 750
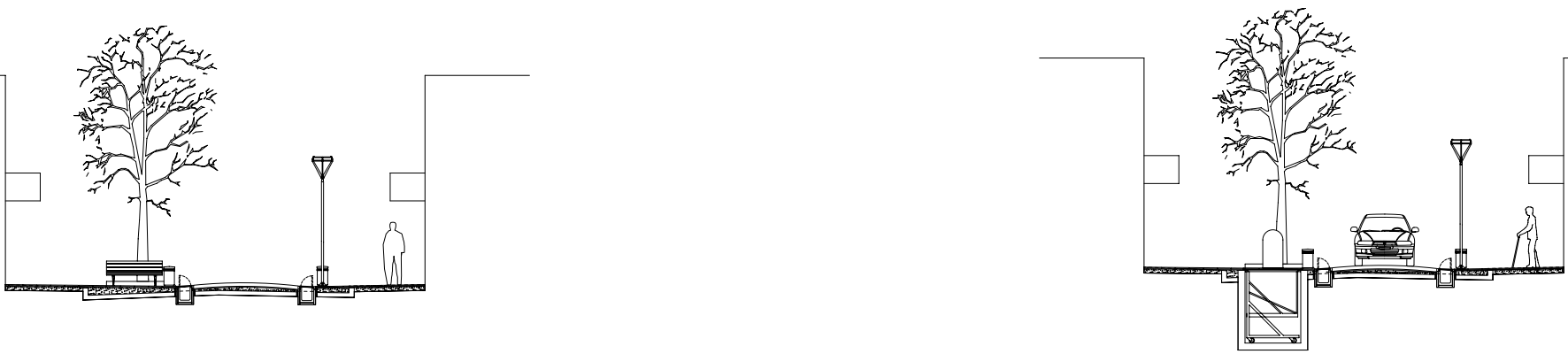

Corte tipo AA, calle Constitución

E. 1: 250 

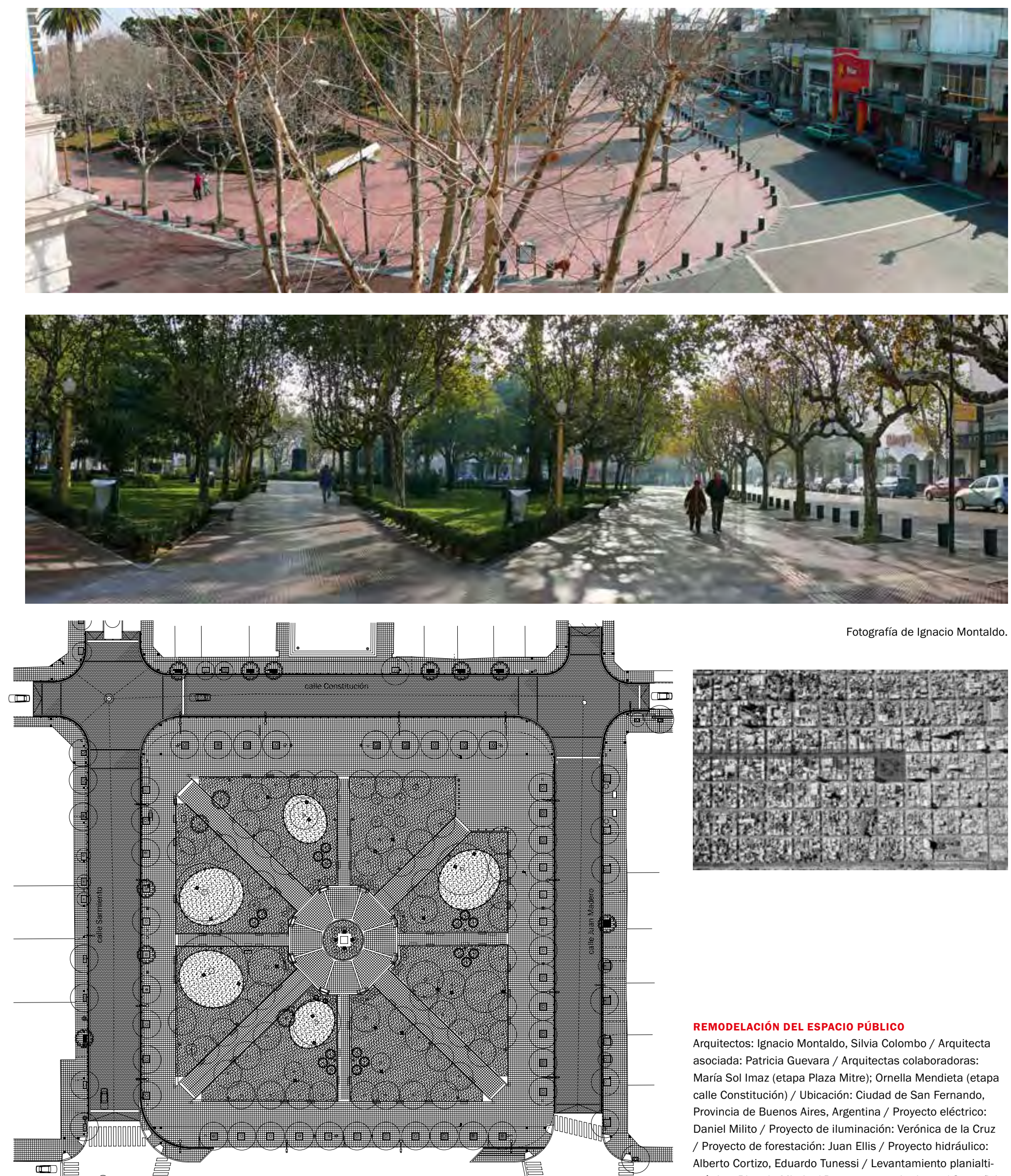

REMODELACIÓN DEL ESPACIO PÚBLICO

Arquitectos: Ignacio Montaldo, Silvia Colombo / Arquitecta sociada: Patricia Guevara / Arquitectas colaboradoras: María Sol Imaz (etapa Plaza Mitre); Ornella Mendieta (etapa calle Constitución) / Ubicación: Ciudad de San Fernando, Provincia de Buenos Aires, Argentina / Proyecto eléctrico: Daniel Milito / Proyecto de iluminación: Verónica de la Cruz / Proyecto de forestación: Juan Ellis / Proyecto hidráulico: Alberto Cortizo, Eduardo Tunessi / Levantamiento planialtimétrico: Ricardo Miorin / Presupuesto: sin datos / Superficie de terreno: $12.000 \mathrm{~m}^{2}$ (etapa plaza Mitre); $10.500 \mathrm{~m}^{2}$ (etapa calle Constitución) / Año de proyecto: 2005 -2006 (etapa plaza Mitre); 2009 (etapa calle Constitución) / Año de construcción: 2006 - 2007 (etapa plaza Mitre); 2010 - 2013 (etapa calle Constitución) / Fotografía: Albano García.

Bibliografía sugerida

MONTALDO, Ignacio. "Plaza Sarmiento, San Fernando, Argentina”. Revista $A R Q$ № 74 Ocio. Ediciones ARQ, Santiago, abril de 2010. 